\title{
Evaluation of Time Domain and Spectral Domain Optical Coherence Tomography in the Measurement of Diabetic Macular Edema
}

\author{
Farzin Forooghian ${ }^{1}$, Catherine Cukras ${ }^{1}$, Catherine B. Meyerle ${ }^{1}$, Emily Y. Chew ${ }^{1}$, and Wai T. \\ Wong $^{2}$ \\ ${ }^{1}$ Division of Epidemiology and Clinical Research, National Eye Institute, National Institutes of Health, \\ Bethesda, Maryland ${ }^{2}$ Office of the Scientific Director, National Eye Institute, National Institutes of Health, \\ Bethesda, Maryland
}

\begin{abstract}
Purpose-To evaluate macular thickness and volume measurements and their intrasession repeatability in two optical coherence tomography (OCT) systems: the Stratus OCT, a time domain system, and the Cirrus HD-OCT, a spectral domain system (both by Carl Zeiss Meditec, Inc., Dublin, $\mathrm{CA}$ ), in the context of diabetic macular edema (DME).

Methods-Thirty-three eyes of 33 diabetic patients with clinically significant macular edema (CSME) were scanned in a single session by a single operator on both OCT systems. Macular thickness measurements of nine standard macular subfields and total macular volume were obtained and analyzed. Bland-Altman plots were constructed to assess agreement in macular measurements. Intraclass correlation coefficients (ICCs), coefficients of repeatability $\left(\mathrm{CR}_{\mathrm{W}}\right)$, and coefficients of variation $\left(\mathrm{CV}_{\mathrm{W}}\right)$ were used to assess intrasession repeatability.
\end{abstract}

Results-Macular thickness in nine retinal subfields and macular volume were significantly higher in the Cirrus HD-OCT system compared with the Stratus OCT system. Subfield thickness and total volume measurements, respectively, were 30 to $55 \mu \mathrm{m}$ and $3.2 \mathrm{~mm}^{3}$ greater for the Cirrus HD-OCT system compared with the Stratus OCT system. Both Stratus OCT and Cirrus HD-OCT systems demonstrated high intrasession repeatability, with overlapping ranges for $\mathrm{CR}_{\mathrm{W}}, \mathrm{CV}_{\mathrm{W}}$, and ICC. Repeatability measures $\left(\mathrm{CR}_{\mathrm{W}}\right.$ and $\left.\mathrm{CV}_{\mathrm{W}}\right)$ differed significantly between systems in only one of nine subfields (outer temporal subfield).

Conclusions-Absolute measures of macular thickness and volume in patients with DME differed significantly in magnitude between the Stratus OCT and Cirrus HD-OCT systems. However, both OCT systems demonstrated high intrasessional repeatability. Although the two systems may not be used interchangeably, they appear equally reliable in generating macular measurements for clinical practice and research.

Optical coherence tomography (OCT) evaluation of macular structure is important in the diagnosis and monitoring of patients with diabetic macular edema (DME). Automated measurements of retinal thickness and volume in the macula from retinal tomograms using image-processing software have been useful in observing patients longitudinally in clinical

Corresponding author: Wai T. Wong, Office of the Scientific Director, National Eye Institute, National Institutes of Health, 7 Memorial Drive, Building 7, Room 217, Bethesda, MD 20892; wongw@nei.nih.gov..

Disclosure: F. Forooghian, None; C. Cukras, None; C.B. Meyerle, None; E.Y. Chew, None; W.T. Wong, None 
practice and making comparisons in clinical trials. To date, time domain OCT using the Stratus OCT (Carl Zeiss Meditec, Inc., Dublin, CA) has been the most widely used technique for obtaining macular measurements ${ }^{1-5}$ and has been shown in previous studies to generate measurements with high repeatability. ${ }^{6-9}$ This instrument acquires images at a rate of 400 axial scans per second, with an axial resolution of $10 \mu \mathrm{m}$. Recently, a new class of OCT instruments employing spectral (Fourier) domain technology has been developed. One such instrument that is currently commercially available is the Cirrus HD-OCT (Carl Zeiss Meditec, Inc.). Although both time domain and spectral domain OCT use the same basic working principles, the scan rate of spectral domain OCT is at least 20,000 axial scans per second, with an improved axial resolution of $5 \mu \mathrm{m}$.

Both the Stratus OCT and the Cirrus HD-OCT systems employ intrinsic software algorithms to calculate retinal thicknesses averaged across standardized subfields in the macula. Data for thickness calculations are collected from an array of A-scans distributed across the macula. This array differs between the Stratus OCT and Cirrus HD-OCT systems. In the Cirrus HDOCT system, significantly more A-scans are used, and these are evenly distributed over the scanned area. The Stratus OCT employs fewer A-scans, and these are more heavily weighted toward the center of the scan. The Stratus OCT system corrects for this effect by extrapolating across areas not sampled; but, depending on the nature of the macular anatomy in those areas, quantitative differences in macular thickness computations may arise between the OCT systems. Also, although both OCT software systems perform automated delineations of retinal boundaries, each system uses different anatomic landmarks in the specification of the outer retinal boundary (information from the manufacturer, Carl Zeiss Meditec, Inc.). These differences may contribute to a different absolute value for thickness measurements, and may also affect how retinal boundaries are reliably delineated between the two OCT systems.

For both clinical practice and in the conduct of clinical trials, the repeatability of OCT measurements has an important impact on how the data may be interpreted. The multiple differences between the time domain Stratus OCT and the spectral domain Cirrus HD-OCT systems may influence how repeatable macular measurements are in the context of DME where there may be considerable deviation from normal anatomy. Spectral domain OCT has been demonstrated to improve visualization of retinal structures in various macular diseases. ${ }^{10-12}$ The reliability of macular measurements obtained by using spectral domain OCT, however, has not been determined. Furthermore, a comparison of the reliability of these two OCT technologies in the determination of macular measurements has not been performed. The purpose of this study was to evaluate and compare the reliability of macular measurements obtained using time domain versus spectral domain OCT in patients with DME. The results of this study will provide information on how OCT macular measurements using both time domain and spectral domain technology can be compared and will further our understanding of how different modes of data capture and analysis in OCT systems can influence the measurements.

\section{Methods}

\section{Subjects}

Eligibility criteria for entry into the study included clinical evidence of diabetic retinopathy with clinically significant macular edema (CSME) as defined by the Early Treatment Diabetic Retinopathy Study (ETDRS). ${ }^{13}$ Eyes with significant media opacities that can result in poor OCT signal were excluded. Eyes with other conditions that can cause macular thickening such as venous occlusion, epiretinal membrane, and/or vitreomacular traction were also excluded. In patients with bilateral macular edema qualifying for inclusion, only one study eye was chosen by a randomized process. The study was performed with informed patient consent and 
was conducted under a protocol approved by the local institutional review board (IRB) and in accordance with the ethical standards stated in the 1964 Declaration of Helsinki.

\section{Optical Coherence Tomography}

The two scanners used in this study were the Stratus OCT (software ver. 5.0), a time domain OCT instrument, and the Cirrus HD-OCT (software ver. 2.0), a spectral domain OCT instrument (both commercially available from Carl Zeiss Meditec, Inc.). Information on scanning modes and image analyses were obtained from the manufacturer. Scanning with the Stratus OCT was performed by using the fast macular thickness map protocol, which acquires six evenly spaced 6-mm radial lines, consisting of 128 A-scans per line, intersecting at the fovea (total of 768 sampled points) within a scan time of 1.9 seconds. Scanning with the Cirrus HD-OCT was performed with the $512 \times 128$ scan pattern where a $6 \times 6-\mathrm{mm}$ area on the retina is scanned with 128 horizontal lines, each consisting of $512 \mathrm{~A}$-scans per line (total of 65,536 sampled points) within a scan time of 2.4 seconds. A schematic providing a comparison of the Stratus OCT and Cirrus HD-OCT scanning patterns is shown in Figure 1.

Each study eye was pharmacologically dilated with $2.5 \%$ phenylephrine hydrochloride and $1 \%$ tropicamide and scanned sequentially on both instruments in the same session in random order. All scans were performed by a single experienced technician who is certified by image reading centers for OCT imaging in multicenter clinical trials. Three high-quality scans were obtained with each instrument. These were defined as scans with a signal strength $\geq 6$ that exhibit correct delineation of the retina layers as detected automatically by the intrinsic software segmentation algorithm and are without image artifacts caused by eye movement and pupillary shadowing. Six scanning attempts per instrument per patient were made, and the first three scans meeting the criteria for high quality were used for image analysis. The macular grid was centered on the intrinsic fixation target during OCT scanning, and decentration of the grid by the technician to attempt to center the grid on the fovea was not allowed.

OCT data were processed on both instruments by using the software versions mentioned earlier. Intrinsic retinal segmentation algorithms were used to define an internal and an external retinal layer position from which retinal thickness and volume measurements were derived. In the computational software, retinal thicknesses in both instruments are averaged within nine retinal subfields in a 6-mm-diameter circle centered on the fovea, as defined in the Age Related Eye Disease Study (AREDS). ${ }^{14}$ The standard retinal subfields are central, inner superior, inner nasal, inner inferior, inner temporal, outer superior, outer nasal, outer inferior, and outer temporal. These subfields are depicted in Figure 2. Overall macular volume over the entire grid area was also obtained from the computational software output.

\section{Statistical Analysis}

Based on the results of a recent study of macular measurements obtained by the Stratus OCT,

7 the mean central thickness in patients with CSME was reported to be $404 \mu \mathrm{m}$, with a standard deviation of $108 \mu \mathrm{m}$. Using a two-sided level of significance $(\alpha)$ of 0.05 and a power $(1-\beta)$ of 0.99 , a minimum sample size of 33 was calculated as being necessary for detecting a $20 \%$ difference in mean macular thickness measurements with a paired experimental design. Macular thickness readings for each of the retinal subfields were obtained for each patient on both OCT scanners, and Bland-Altman plots ${ }^{15}$ (Prism, ver. 4; GraphPad, San Diego, CA) were constructed to compare and assess agreement in macular measurements between the Stratus OCT and Cirrus HD-OCT systems.

To assess and compare the intrasession repeatability of the two scanners, the intrasession within-subject standard deviation $\left(\mathrm{S}_{\mathrm{W}}\right)$, coefficient of repeatability $\left(\mathrm{CR}_{\mathrm{W}}\right)\left(1.96 \times \mathrm{S}_{\mathrm{W}}\right)$, coefficient of variation $\left(\mathrm{CV}_{\mathrm{W}} ; 100 \% \times \mathrm{S}_{\mathrm{W}} /\right.$ overall mean $)$, and intraclass correlation coefficient 
(ICC) were calculated for each scanner (SPSS, ver. 15.0; SPSS, Chicago, IL). ${ }^{7,16}$ For the ICC calculation, a two-way mixed-effects model for measures of absolute agreement and single ratings was used. ICC values of Stratus OCT and Cirrus HD-OCT were compared by examination of respective confidence intervals (CIs). Pearson correlation analysis was performed for the mean subfield measurements for each eye and their respective $\mathrm{CR}_{\mathrm{W}}$ and $\mathrm{CV}_{\mathrm{W}}$ values (Prism, ver. 4; GraphPad). Pair-wise comparisons of retinal measurements $\mathrm{CR}_{\mathrm{W}}$ and $\mathrm{CV}_{\mathrm{W}}$ between Stratus OCT and Cirrus HD-OCT systems (using the two-tailed paired $t$-test with Bonferroni adjustment for multiple comparisons) were performed (Prism, ver. 4; GraphPad). Pair-wise comparisons between included and excluded patient characteristics was performed by unpaired two-tailed $t$-test, incorporating the Bonferroni adjustment and Welch's correction (Prism, ver. 4; GraphPad).

\section{Results}

A total of 44 eligible eyes of 44 patients were prospectively entered into the scanning protocol, and 33 eyes of 33 patients were included in the subsequent analysis. Eleven eyes were not analyzed, as their scans did not meet the criteria for being of high quality. Of these, five eyes had an insufficient number of scans $(<3)$ with suprathreshold signal strength $(\geq 6)$ on only the Stratus OCT, while three other eyes were removed for the same reason on both Stratus OCT and Cirrus HD-OCT systems. In three other eyes, errors in the proper delineation of retinal layers were found on both systems despite having suprathreshold signal strength. Table 1 shows the characteristics of both the included and excluded eyes. Eyes in the two categories were not significantly different in mean visual acuity, central subfield macular thickness, or patient age. As expected, the excluded eyes differed from the included eyes with regard to signal strength on both OCT scanners. Varied clinical patterns of DME were seen in study eyes, with the patterns occurring in included and excluded eyes alike. Most of the study eyes had a focal pattern of DME with minimal exudates. Among the excluded eyes $(n=11)$, two had a diffuse pattern of DME, whereas one had significant hard exudates. Among the included eyes $(n=$ 33), five had a diffuse pattern of DME, and another seven had significant hard exudates.

Mean measurements for macular thickness in each of the nine standard subfields, as well as total macular volume, obtained using both OCT scanners is shown in Table 2. For central macular thickness, the two scanners produced measurements that agreed poorly with each other, as demonstrated by the Bland-Altman plot (Fig. 3A), whose $95 \%$ limits of agreement were between -137 and $31 \mu \mathrm{m}$. The Cirrus HD-OCT measurements were significantly higher than those from the Stratus OCT system $(P<0.005)$ in pair-wise comparisons, being on average $53 \mu \mathrm{m}$ higher. Pair-wise comparisons for all the other retinal subfields revealed a significantly higher measurement by Cirrus OCT (all comparisons, $P<0.005$ ), with their respective BlandAltman plots also revealing poor agreement (data not shown). These thickness differences were mostly lower than that found in the central subfield, with inner superior and outer superior subfields having the least difference. With respect to macular volume, the overall volume computed by the two scanners also agreed poorly (Fig. 3B), with $95 \%$ limits of agreement between -3.8 and $-2.7 \mathrm{~mm}^{3}$. Pair-wise comparisons show a significantly larger overall macular volume $(P<0.005)$ measured by the Cirrus HD-OCT system, being on average $3.2 \mathrm{~mm}^{3}$ higher than that measured by the Stratus OCT system.

The intrasession repeatability of the two scanners was calculated and compared. Table 3 shows the ICCs along with their respective CIs, whereas Table 4 shows the $\mathrm{CR}_{\mathrm{W}}$ and $\mathrm{CV}_{\mathrm{W}}$ values $( \pm$ SD), for all retinal subfields and macular volume. Both Stratus OCT and Cirrus OCT HDOCT scanning systems demonstrated high repeatability on measures of retinal subfield thickness in all subfields, with respective $\mathrm{CR}_{\mathrm{W}}$ falling below 19 and $21 \mu \mathrm{m}$, respective $\mathrm{CV}_{\mathrm{W}}$ below $3.2 \%$ and $2.9 \%$, and respective ICCs exceeding 0.92 and 0.83 . Furthermore, measures of repeatability for both OCT systems demonstrated overlapping ranges. Overall macular 
volume measurements showed similar high-repeatability indices for both systems. Comparisons of these measures did not reveal any broad or consistent differences in repeatability between the systems. The only parameter for which measures of repeatability were significantly different was in the $\mathrm{CR}_{\mathrm{W}}$ and the $\mathrm{CV}_{\mathrm{W}}$ for the outer temporal macular thickness subfield. In this one particular subfield, a higher repeatability was found for the Cirrus HD-OCT system than for the Stratus OCT system.

We also examined whether measures of repeatability of the OCT scanning systems were associated with overall macular thickness. We found that for many retinal subfields the coefficients of repeatability $\left(\mathrm{CR}_{\mathrm{W}}\right.$ or $\left.\mathrm{CV}_{\mathrm{W}}\right)$ correlated positively with macular thickness, indicating that in these fields repeatability of thickness measurements decreased as macular thickness increased. This correlation was found in five of nine retinal subfields with the Stratus OCT and in three of nine with the Cirrus HD-OCT (Table 5). The inner and outer nasal subfields were shared between the two systems as subfields in which repeatability decreased as a function of increasing macular thickness.

\section{Discussion}

In this study of patients with DME, we observed that the Cirrus HD-OCT generated significantly higher values for macular thickness in all nine standard subfields. In addition, total macular volume measurements were significantly higher with the Cirrus HD-OCT than with the Stratus OCT. This discrepancy can be readily explained by the differences in retinal segmentation algorithms used by the Stratus OCT and Cirrus HD-OCT systems. The Cirrus HD-OCT segmentation algorithm identifies the thickness of the retina from the retinal pigment epithelium (RPE) to the inner limiting membrane (ILM), while the Stratus OCT segmentation algorithm identifies the thickness of the retina based on the distance between the ILM and junction of the outer segments (OS) and inner segments (IS) of the photoreceptors (Fig. 4). Thus, the Cirrus HD-OCT system would be expected to give macular measurements that are larger than those obtained by the Stratus system. The difference in thickness measurements between the Cirrus HD-OCT and Stratus OCT systems should correspond to the length of photoreceptor outer segments within the macula. We observed that the mean difference between macular thickness measurements obtained by the Cirrus HD-OCT and Stratus OCT systems in the various subfields ranged from 30 to $55 \mu \mathrm{m}$. These observations are consistent with the $50-\mu \mathrm{m}$ length of the outer segments of human photoreceptors. ${ }^{17}$ In this study, there was no clear superiority in the ability of the two OCT systems to avoid segmentation errors. Of the 44 eyes scanned, only 3 with suprathreshold signal strength $(\geq 6)$ were not included in the analysis due to segmentation errors. These errors were found in both systems, however, for all three eyes.

Bland and Altman ${ }^{15}$ have described that two methods of measurement may be used interchangeably if the $95 \%$ limits of agreement between the two instruments falls into a clinically acceptable range. We observed that the $95 \%$ limits of agreement between the measurements obtained from the Stratus OCT and Cirrus HD-OCT system were large. Based on the Bland-Altman 95\% limits of agreement, the difference in macular thickness measurements between the two systems could be as great as $168 \mu \mathrm{m}-$ a difference that is clinically unacceptable. Thus, macular thickness measurements obtained with the Cirrus HDOCT cannot be used interchangeably with those obtained with the Stratus OCT. Similar poor agreement was observed in macular volume measurements between the two scanners.

In this study, we measured and compared the repeatability indices of the Stratus OCT and Cirrus HD-OCT systems within the same group of diabetic patients. For OCT scans obtained by the same experienced operator with adequate signal strength and accurate segmentation, both systems produced measures that reflected high repeatability. The repeatability of the 
Stratus OCT in measuring DME has been evaluated, 6,7 and although different methods were used, the repeatability measures are comparable to the ones obtained in this study. The repeatability of the Cirrus HD-OCT system in measuring DME had not been evaluated prior to our current study. In a direct comparison of repeatability measures, our results did not demonstrate a broad and significant difference in repeatability of macular thickness measures between the Stratus OCT and Cirrus HD-OCT. A significant difference in repeatability measures was found in only one of nine retinal subfields (outer temporal) for two of the three measures used $\left(\mathrm{CR}_{\mathrm{W}}\right.$ and $\left.\mathrm{CV}_{\mathrm{W}}\right)$. The fact that repeatability was only different in the outer temporal subfield as opposed to any other subfield is probably due to chance alone, as there is no physiological or anatomic reason to explain why macular thickness measurements in this subfield in particular should be more repeatable. Our comparison of the two systems did not involve a large patient population and was only powered to detect differences greater than $20 \%$ with $99 \%$ power. It is possible that smaller differences in repeatability between the two systems may exist and may have been missed in our analysis. However, from a practical standpoint, these smaller reliability differences may have a limited impact on clinical care and the design of clinical trials.

A priori, it may have been expected that the Stratus OCT and the Cirrus HD-OCT systems would have different measures of repeatability. The scanning rates and patterns used differ significantly, with the Cirrus HD-OCT performing many more sampling scans in an uniform density across the imaging field than the Stratus OCT. Both OCT systems estimate the mean thickness in each of these subfields based on an extrapolation from sampled data points. In the context of DME, in which retinal thickness in each retinal subfield may vary significantly from normal values, it may be expected that a higher sampling density, especially in the outer retinal subfields, may confer a higher repeatability on the Cirrus HD-OCT compared with the Stratus OCT. These intrinsic differences, however, did not result in a measurable difference in repeatability. One explanation for this observation may be that extrinsic factors, such as patient fixation and the ability to consistently place the macular grid over the same points during each scan, may have more of an influence on reliability of OCT measurements than data sampling capability and extrapolation by software. Patient fixation would be expected to be suboptimal in conditions such as DME where increased macular thickness could lead to poor fixation. Indeed, we observed that increased macular thickness correlated with poor repeatability in several subfields in both OCT systems. As eyes with delineation errors were excluded from the analysis, it is not likely that an increasing error rate in retinal delineation with increasing macular thickness is contributory to the relationship found in the study.

In addition to patient fixation, the number of scans per unit area may affect the repeatability of macular measurements. In this study we chose the Cirrus $512 \times 128$-cube protocol over the Cirrus $200 \times 200$-cube protocol because of the higher density of sampling points across the imaging field and thus the lower degree of extrapolation used between points. The alternative comparison that was not performed in this study was to compare the Cirrus HD OCT $200 \times$ 200-cube protocol to the Stratus OCT fast macular thickness scan protocol. Each of the scan lines in the Cirrus HD-OCT $200 \times 200$-cube protocol will have a lower resolution of A-scans but will have a shorter total scan time compared to the Cirrus HD-OCT $512 \times 128$-cube protocol (1.3 seconds compared with 2.4 seconds). Future studies may be needed to demonstrate that scanning time rather than scanning density is the more important determinant for increased reliability in generating macular thickness measurements in OCT imaging.

In summary, we found that Cirrus HD-OCT provided significantly higher macular thickness and volume measurements than did Stratus OCT. The two OCT instruments cannot be used interchangeably for the measurement of macular thickness and volume. We did not observe a significant difference in intrasession repeatability between these two systems when using standard scanning protocols. Faster scan rates and increased scanned points may not yield more 
reliable measurements in patients with DME, in which the main determinant of reliability may be the patient's fixation. Although the higher image resolution afforded by Cirrus HD-OCT over Stratus OCT may show the former to be superior in the visualization of macular structure, the similar reliability of these systems makes them comparable in the setting of measurement of macular thickness and volume parameters in patients with DME.

\section{Acknowledgments}

The authors thank Gregory Short for performing the OCT scans and Elvira Agrón for assistance with statistical analysis.

Supported by the National Eye Institute Intramural Research Program.

\section{References}

1. Jaffe GJ, Caprioli J. Optical coherence tomography to detect and manage retinal disease and glaucoma. Am J Ophthalmol 2004;137:156-169. [PubMed: 14700659]

2. Otani T, Kishi S, Maruyama Y. Patterns of diabetic macular edema with optical coherence tomography. Am J Ophthalmol 1999;127:688-693. [PubMed: 10372879]

3. Haouchine B, Massin P, Tadayoni R, et al. Diagnosis of macular pseudoholes and lamellar macular holes by optical coherence tomography. Am J Ophthalmol 2004;138:732-739. [PubMed: 15531306]

4. Sanchez-Tocino H, Alvarez-Vidal A, Maldonado MJ, et al. Retinal thickness study with optical coherence tomography in patients with diabetes. Invest Ophthalmol Vis Sci 2002;43:1588-1594. [PubMed: 11980878]

5. Politoa A, Napolitano MC, Bandello F, et al. The role of optical coherence tomography (OCT) in the diagnosis and management of retinal angiomatous proliferation (RAP) in patients with age-related macular degeneration. Ann Acad Med Singapore 2006;35:420-424. [PubMed: 16865194]

6. Massin P, Vicaut E, Haouchine B, et al. Reproducibility of retinal mapping using optical coherence tomography. Arch Ophthalmol 2001;119:1135-1142. [PubMed: 11483079]

7. Polito A, Del Borrello M, Isola M, et al. Repeatability and reproducibility of fast macular thickness mapping with Stratus optical coherence tomography. Arch Ophthalmol 2005;123:1330-1337. [PubMed: 16219723]

8. Danis RP, Fisher MR, Lambert E, et al. Results and repeatability of retinal thickness measurements from certification submissions. Arch Ophthalmol 2008;126:45-50. [PubMed: 18195217]

9. Krzystolik MG, Strauber SF, Aiello LP, et al. Reproducibility of macular thickness and volume using Zeiss optical coherence tomography in patients with diabetic macular edema. Ophthalmology 2007;114:1520-1525. [PubMed: 17353052]

10. Hangai M, Ojima Y, Gotoh N, et al. Three-dimensional imaging of macular holes with high-speed optical coherence tomography. Ophthalmology 2007;114:763-773. [PubMed: 17187861]

11. Ojima Y, Hangai M, Sasahara M, et al. Three-dimensional imaging of the foveal photoreceptor layer in central serous chorioretinopathy using high-speed optical coherence tomography. Ophthalmology 2007;114(12):2197-2207. [PubMed: 17507096]

12. Ahlers C, Michels S, Beckendorf A, et al. Three-dimensional imaging of pigment epithelial detachment in age-related macular degeneration using optical coherence tomography, retinal thickness analysis and topographic angiography. Graefes Arch Clin Exp Ophthalmol 2006;244:1233-1239. [PubMed: 16977431]

13. Early Treatment Diabetic Retinopathy Study research group. Photocoagulation for diabetic macular edema. Early Treatment Diabetic Retinopathy Study report number 1. Arch Ophthalmol 1985;103:1796-1806. [PubMed: 2866759]

14. Davis MD, Gangnon RE, Lee LY, et al. The Age-Related Eye Disease Study severity scale for agerelated macular degeneration: AREDS Report No. 17. Arch Ophthalmol 2005;123:1484-1498. [PubMed: 16286610]

15. Bland JM, Altman DG. Statistical methods for assessing agreement between two methods of clinical measurement. Lancet 1986;1:307-310. [PubMed: 2868172] 
16. Li H, Leung CK, Wong L, et al. Comparative study of central corneal thickness measurement with slit-lamp optical coherence tomography and Visante Optical Coherence Tomography. Ophthalmology 2008;115(5):796-801.e2. [PubMed: 17916376]

17. Hendrickson A, Drucker D. The development of parafoveal and mid-peripheral human retina. Behav Brain Res 1992;49:21-31. [PubMed: 1388798] 

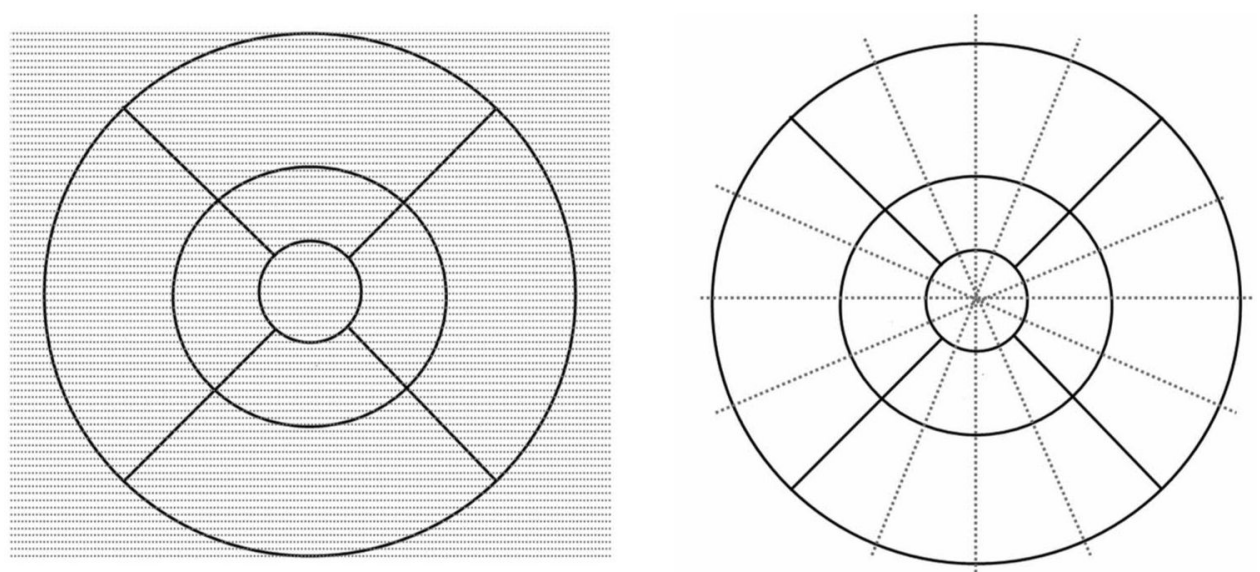

Figure 1.

Systems of retinal sampling used by the Cirrus HD-OCT and Stratus OCT systems (Carl Zeiss Meditec, Inc., Dublin, CA). Shown are the different sampling methods for the Cirrus HD-OCT (left) and the Stratus OCT (right). Cirrus HD-OCT samples significantly more points that are evenly distributed over the macular grid, whereas Stratus OCT samples points along six radial lines that intersect at the center of the macular grid. 


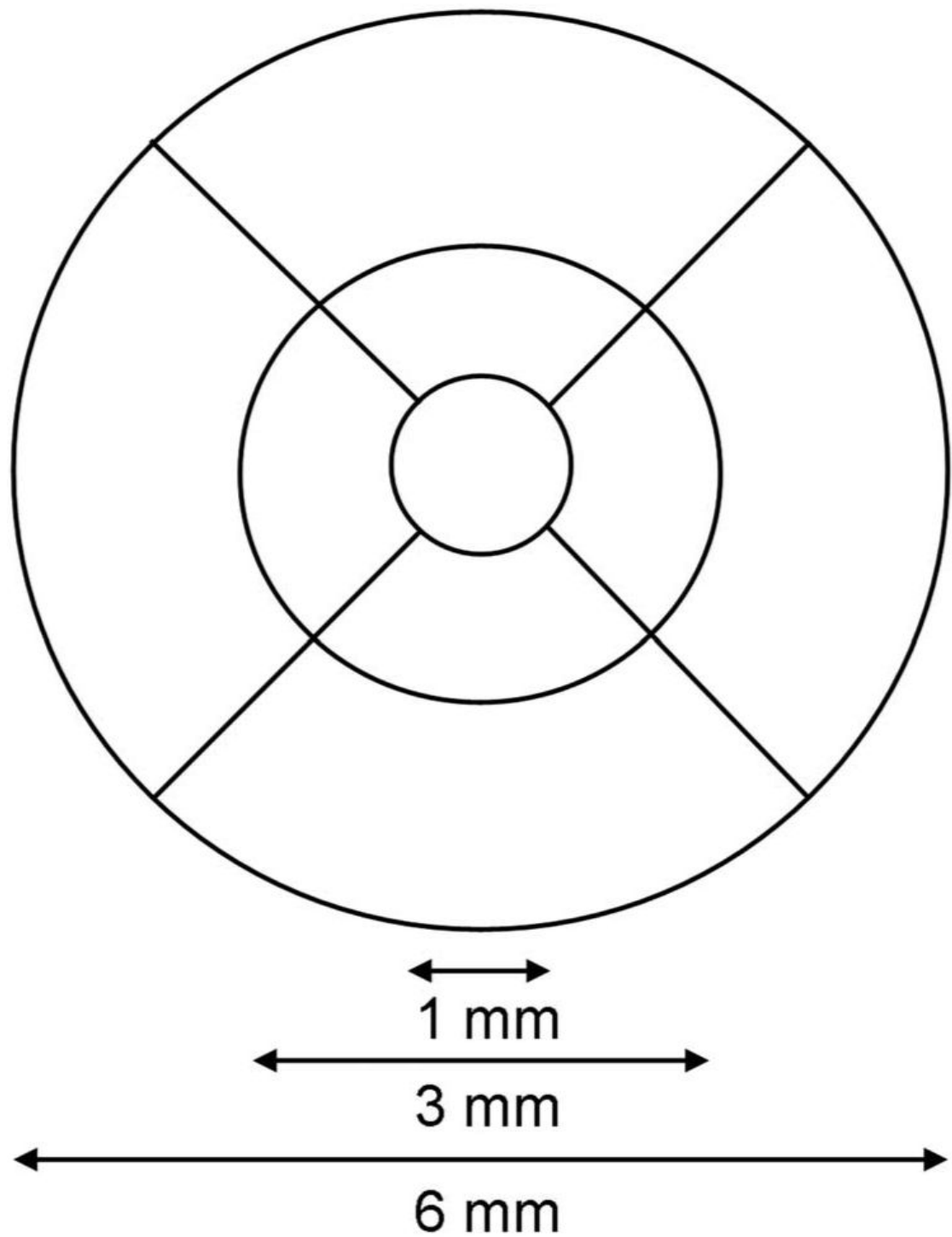

Figure 2.

Macular grid used by both the Cirrus HD-OCT and Stratus OCT systems (Carl Zeiss Meditec, Inc., Dublin, CA). Both systems employ the same 6-mm-diameter grid defined by the AgeRelated Eye Disease Study (AREDS). The inner subfields are bounded by the 3-mm-diameter circle, whereas the central subfield is bounded by the inner-most 1 -mm-diameter circle. 


\section{Central Subfield}

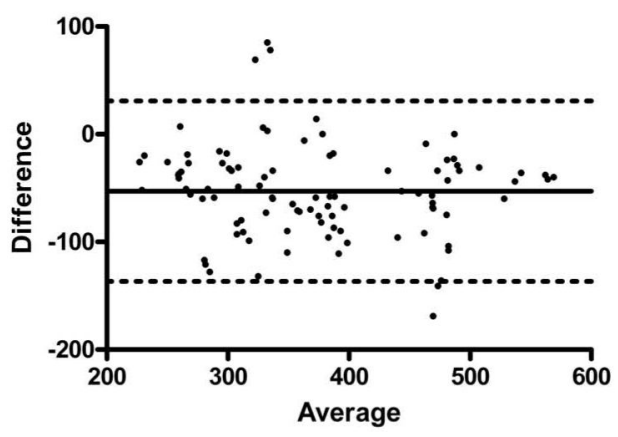

Total Volume

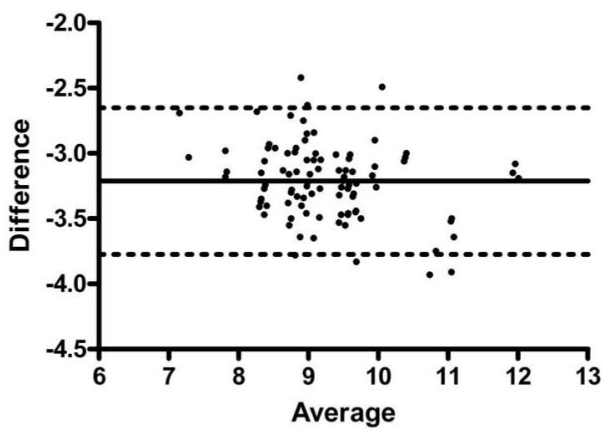

Figure 3.

Representative Bland-Altman plots of macular measurements obtained using Stratus OCT compared to the Cirrus HD-OCT. Bland-Altman plots for central subfield macular thickness (left) and macular volume (right) are shown. Solid lines: the bias (average mean difference); dotted lines: $95 \%$ confidence limits of agreement. 


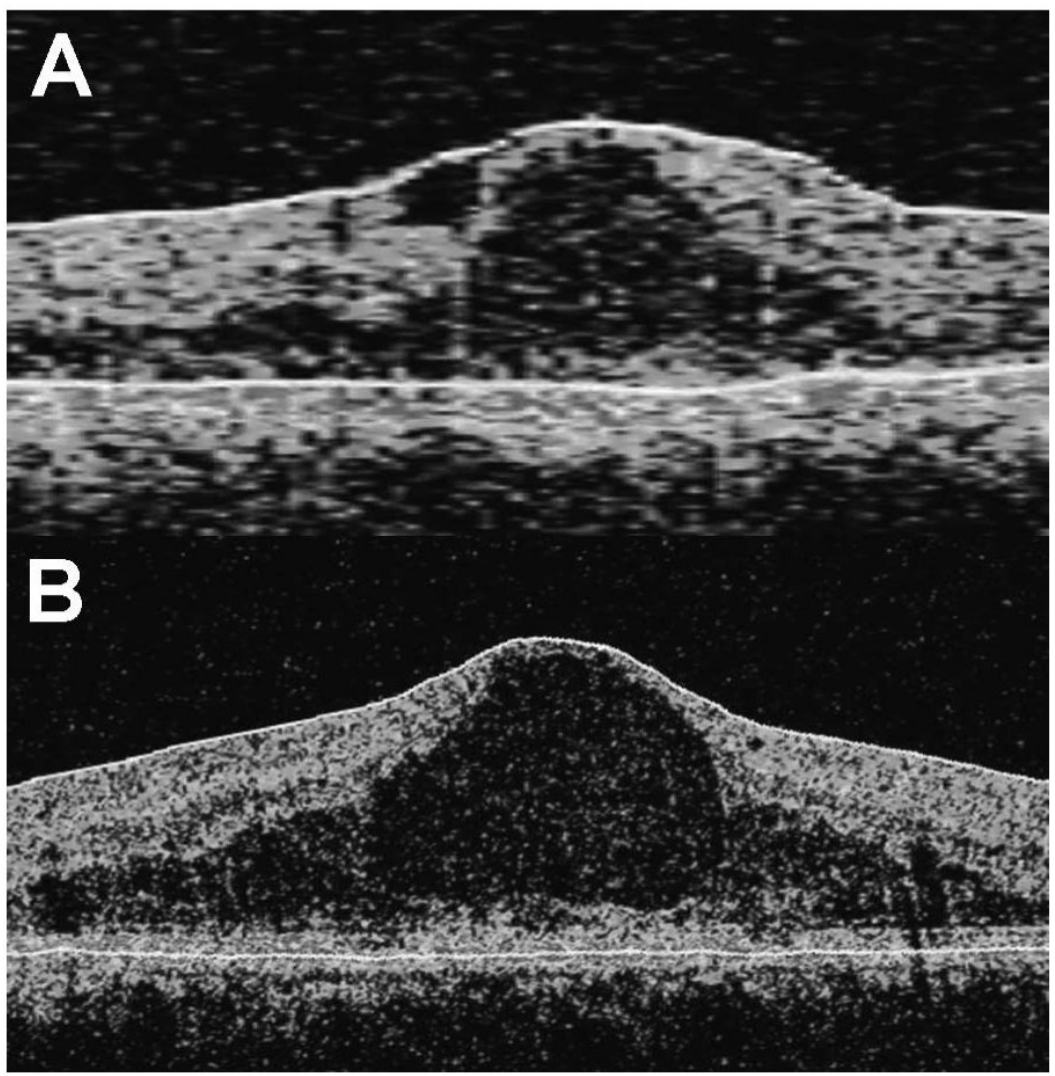

Figure 4.

Differences in delineation of retinal layers by the Stratus OCT compared with the Cirrus HDOCT (Carl Zeiss Meditec, Inc., Dublin, CA). Representative OCT scans obtained using the (A) Stratus OCT and (B) Cirrus HD-OCT from a study eye are shown. Segmentation lines are shown in white. The Cirrus HD-OCT segmentation algorithm identifies the thickness of the retina from the retinal pigment epithelium (RPE) to the inner limiting membrane (ILM), whereas the Stratus OCT segmentation algorithm identifies the thickness of the retina based on the distance between the ILM and junction of the outer segments (OS) and inner segments (IS) of the photoreceptors. 


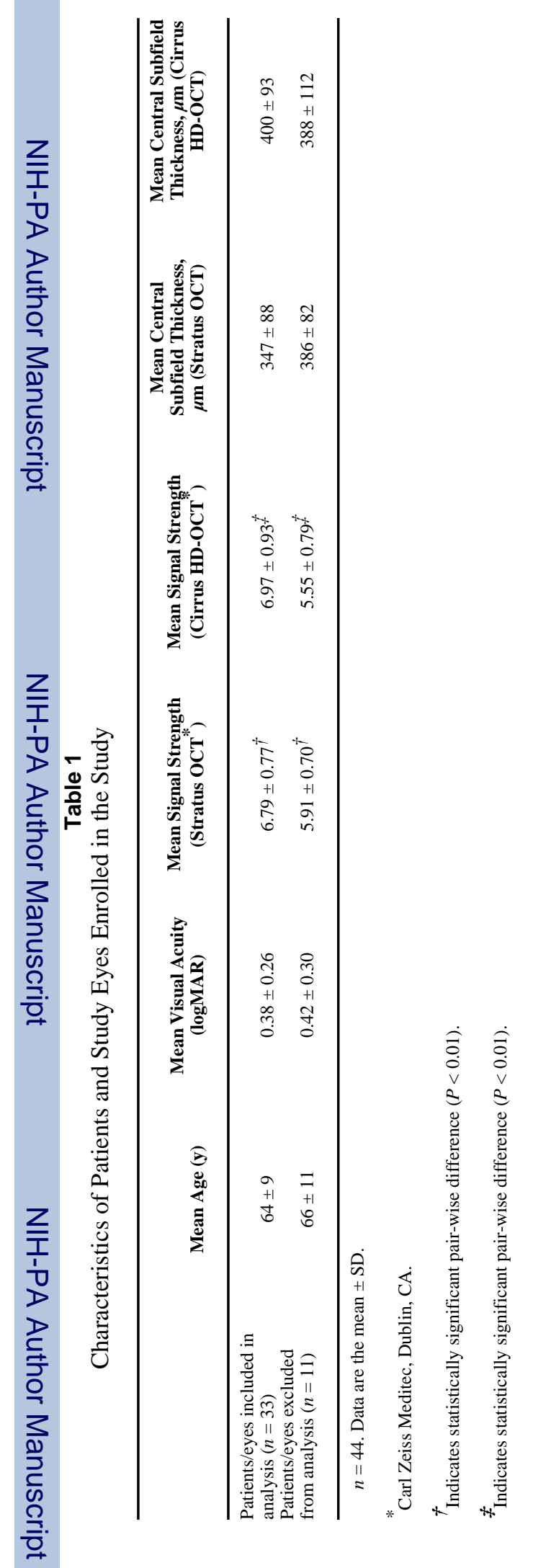




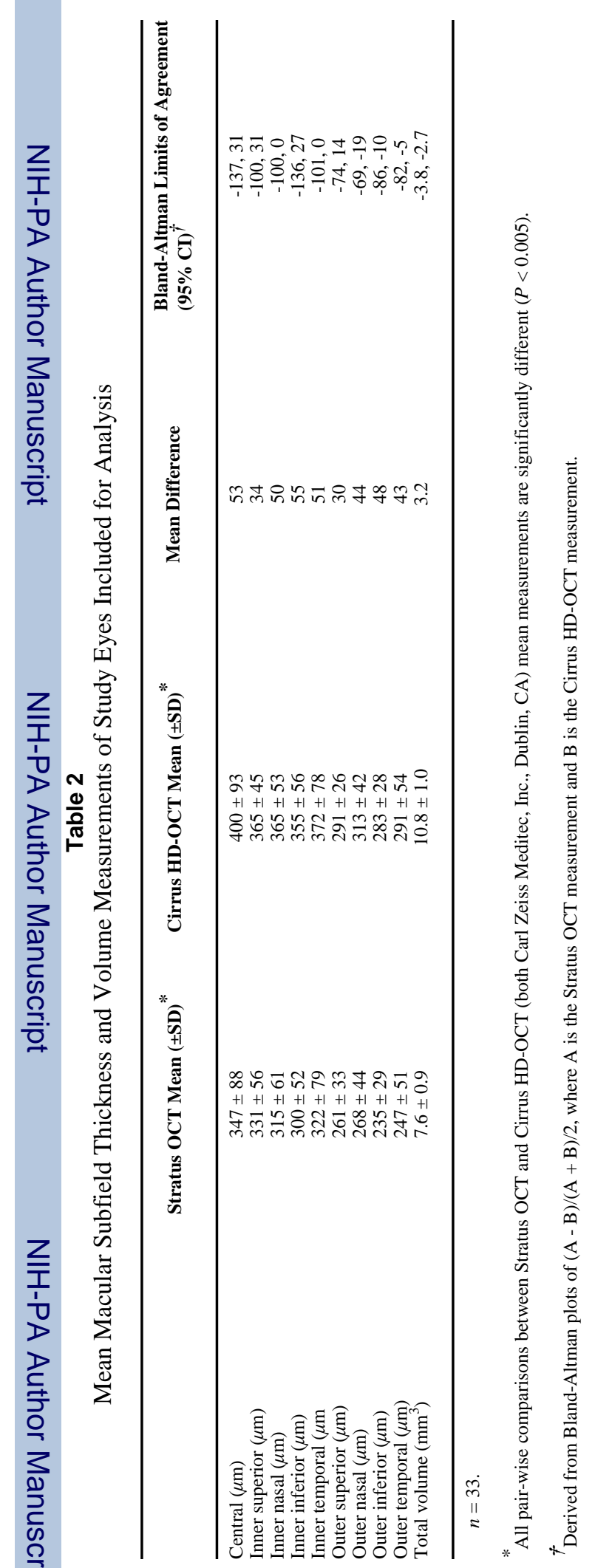




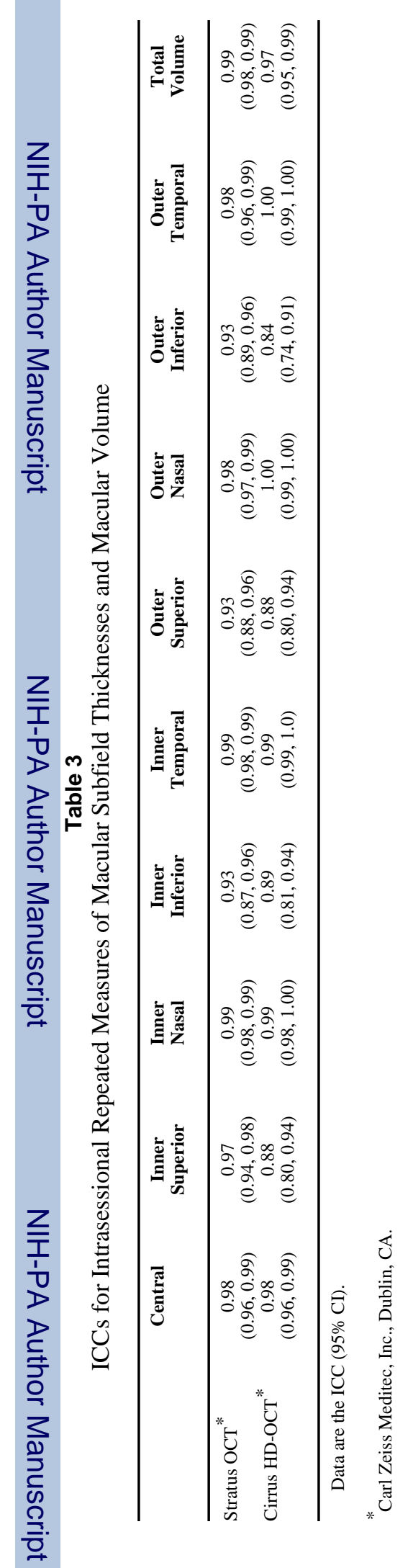




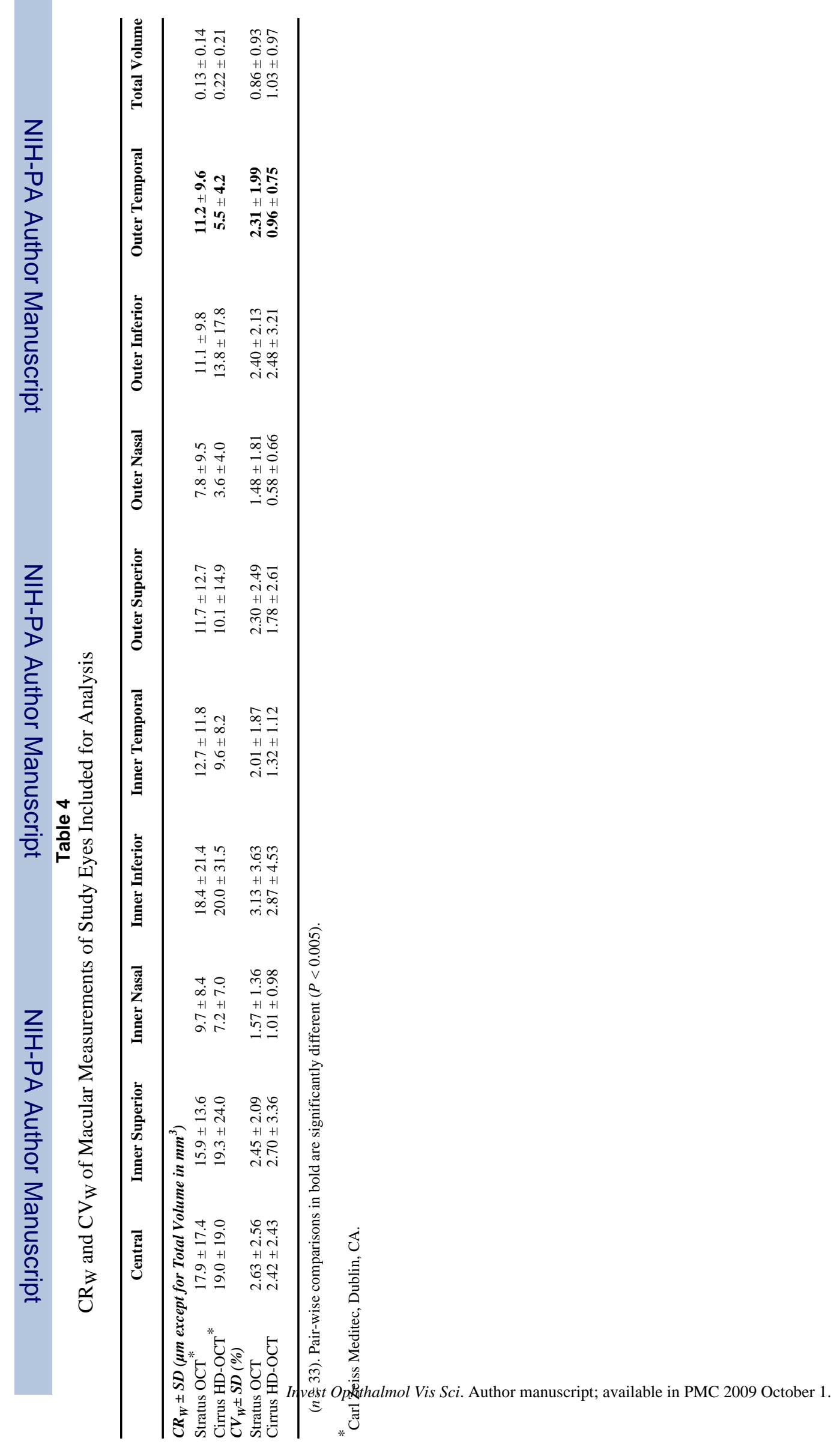




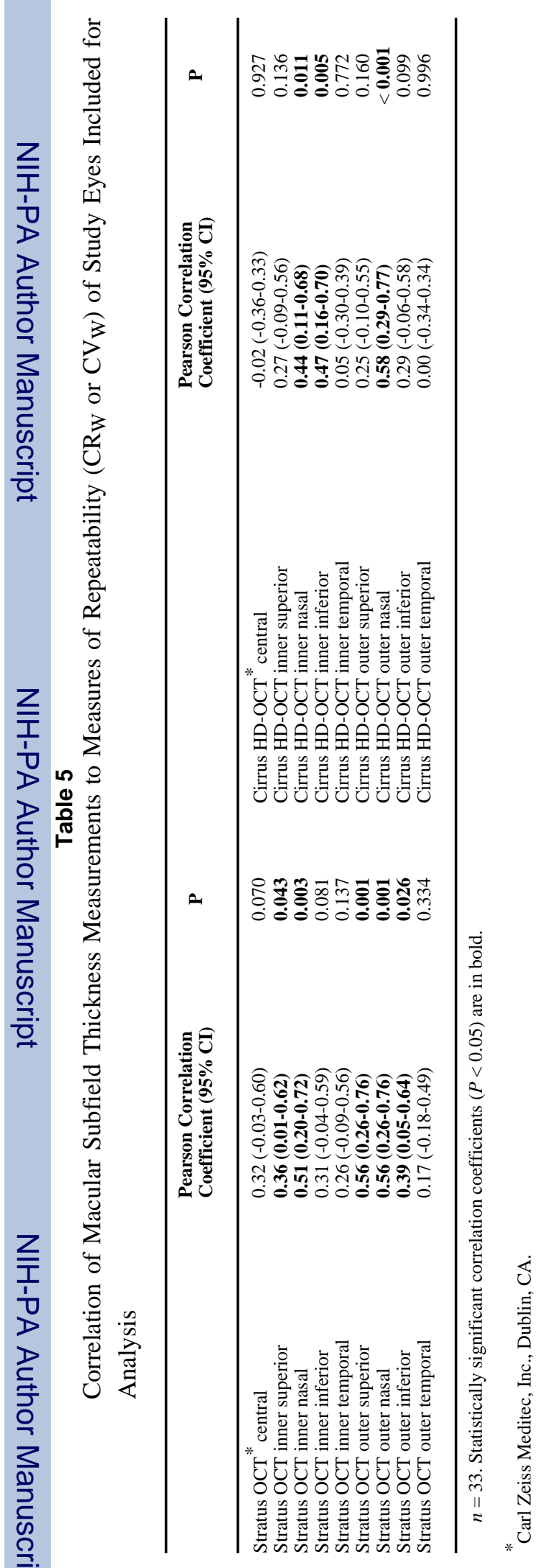

Invest Ophthalmol Vis Sci. Author manuscript; available in PMC 2009 October 1. 